\title{
TINSELSPITAL $\boldsymbol{u}^{b}$ \\ UNIVERSITATSSPITAL BERN \\ BERN UNIYERSITY HOSPITAL

\section{Solitaire $^{\mathrm{TM}}$ With the Intention For Thrombectomy Plus In- travenous t-PA Versus DIRECT Solitaire ${ }^{\mathrm{TM}}$ Stent-retriever Thrombectomy in Acute Anterior Circulation Stroke (SWIFT DIRECT)}

Urs Fischer ${ }^{1}$, Vitor Mendes Pereira ${ }^{2}$, René Chapot ${ }^{3}$, Adnan Siddiqui ${ }^{4}$, Jenny Bressan ${ }^{1}$,

Melanie Schmidhalter ${ }^{1}$, Johannes Kaesmacher ${ }^{1}$, Michael Froehler ${ }^{5}$, Christoph Cognard ${ }^{6}$, Stefanie Lerch ${ }^{1}$, Anthony Furlan ${ }^{7}$, Jeff Saver ${ }^{8}$, Jan Gralla ${ }^{1}$

${ }^{1}$ Bern University Hospital and University of Bern, Bern, Switzerland, ${ }^{2}$ University of Toronto, Canada, ${ }^{3}$ Alfried Krupp Krankenhaus, Essen, Germany, ${ }^{4}$ University at Buffalo, USA, ${ }^{5}$ Vanderbilt University Medical Center, USA, ${ }^{6}$ University of Toulouse, France, ${ }^{7}$ UH Cleveland Medical Center, USA, ${ }^{8}$ University of California, USA

\section{BACKGROUND}

Whether pre-treatment with intravenous thrombolysis (IVT) prior to mechanical thrombectomy (MT) with stent retrievers is beneficial has become a matter of debate. In a patient-level pooled analysis of five randomized controlled studies (HERMES collaboration*) the treatment effect size of MT did not differ between patients receiving IVT and those treated with MT alone. SWIFT DIRECT aims to determine, whether direct MT in patients with proximal vessel occlusion in the anterior circulation is non-inferior to IVT and MT.

\section{METHODS}

The international, multicentre, randomized-controlled, two-arm, open label, blinded endpoint (PROBE) trial SWIFT DIRECT will randomise 404 patients into the experimental arm (direct MT; 202) or control arm (IVT and MT; 202). The trial will only be performed in patients with immediate access to MT $(<90$ minutes from door to groin puncture). Subsequently no drip-and-ship patients will be included.

\section{Main inclusion criteria}

- Informed consent

- $\quad$ Age $\geq 18$ and $<86$ years

- Confirmed large vessel occlusion in the anterior circulation

- National Institute of Health Score Scale (NIHSS) $\geq 8$ and $<30$

- Eligibility for IVT and endovascular therapy

\section{TOP RECRUITING SITES}

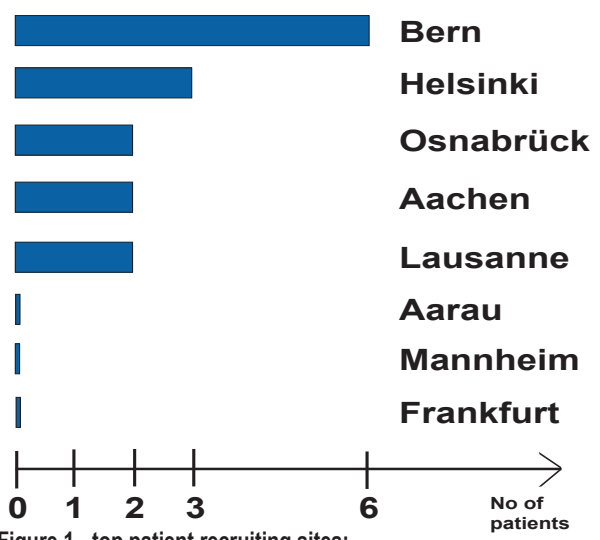

Figure 1 - top patient recruiting sites:

Bern (Switzerland, $n=6$ ), Helsinki (Finland, $n=3$ ), Osnabrück and Aachen (Germany, $n=2$ ), and Lausanne (Switzerland, $n=2$ ). More sites in Austria, Canada, France and Spain will follow soon.

\section{OUTCOMES}

\section{Primary outcome}

- Functional independence (modified Rankin Scale $\leq 2$ ) at 90 days

\section{Secondary outcomes}

- Mortality

- $\quad$ Change in NIHSS score post randomization

- Time to reperfusion

- Rates of successful reperfusion (Thrombolysis in Cerebral Infarction scale $\geq 2 b$ )

- Quality of life

\section{RESULTS}

We started the recruitment in October 2017 (Figure 1 and Figure 2). Within the next weeks multiple centers in Europe and Canada will start to recruit patients.

\section{CONCLUSIONS}

If SWIFT DIRECT is able to prove that direct MT is equally efficacious and safe when compared to current standard treatment (IVT and MT) in stroke patients with large vessel occlusion in the anterior circulation, this would have a relevant impact on future patient management and organization of stroke care. Maintaining a high recruitment rate will be crucial for the successful completion of the trial.

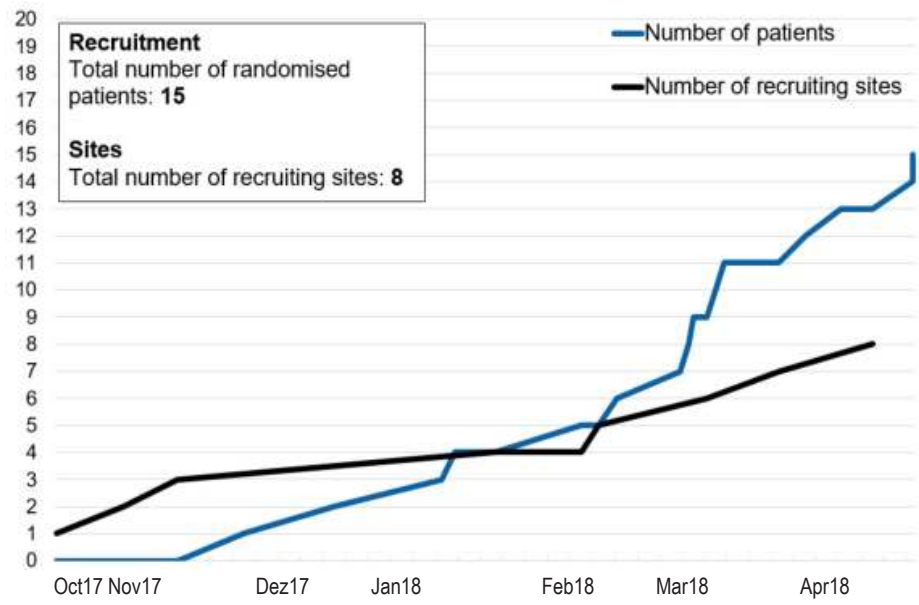

Figure 2 - patient and site recruitment over time period:

15 patients in 8 sites have been randomised from 18.10.2018 to 26.04.2018. 